This item was submitted to Loughborough's Research Repository by the author.

Items in Figshare are protected by copyright, with all rights reserved, unless otherwise indicated.

\title{
Modelling of immiscible liquid-liquid systems by Smoothed Particle Hydrodynamics
}

PLEASE CITE THE PUBLISHED VERSION

https://doi.org/10.1016/j.jcis.2017.08.072

PUBLISHER

(c) Elsevier

VERSION

AM (Accepted Manuscript)

\section{PUBLISHER STATEMENT}

This work is made available according to the conditions of the Creative Commons Attribution-NonCommercialNoDerivatives 4.0 International (CC BY-NC-ND 4.0) licence. Full details of this licence are available at: https://creativecommons.org/licenses/by-nc-nd/4.0/

\section{LICENCE}

CC BY-NC-ND 4.0

\section{REPOSITORY RECORD}

Elekaei, Hamideh, Moein Navvab Kashani, and Mark J. Biggs. 2019. "Modelling of Immiscible Liquid-liquid Systems by Smoothed Particle Hydrodynamics”. figshare. https://hdl.handle.net/2134/27901. 
Modelling of immiscible liquid-liquid systems by Smoothed Particle Hydrodynamics

H. Elekaei Behjatia ${ }^{a}$ M. Navvab Kashania and M.J. Biggs ${ }^{a, b, *}$

a. School of Chemical Engineering, The University of Adelaide, Adelaide, SA, 5005, Australia.

b. School of Science, Loughborough University, Loughborough, LE11 3TU, UK.

*m.biggs@lboro.ac.uk

\begin{abstract}
Immiscible fluid systems are ubiquitous in industry, medicine and nature. Understanding the phase morphologies and intraphase fluid motion is often desirable in many of these situations; for example, this will aid improved design of microfluidic platforms for the production of medicinal formulations. In this paper, we detail a Smoothed Particle Hydrodynamics (SPH) approach that facilitates this understanding. The approach includes surface tension and enforces incompressibility. The approach also allows the consideration of an arbitrary number of immiscible phases of differing viscosities and densities. The nature of the phase morphologies can be arbitrary and change in time, including break-up (which is illustrated) and coalescence. The use of different fluid constitutive models, including non-Newtonian models, is also possible. The validity of the model is demonstrated by applying it to a range of model problems with known solutions, including the Young-Laplace problem, confined droplet deformation under a linear shear field, and a droplet falling under gravity through another quiescent liquid. Results are also presented to illustrate how the SPH model can be used to elucidate the behaviour of immiscible liquid systems.
\end{abstract}

Key Words: Smoothed Particle Hydrodynamics; Incompressible flow; Immiscible liquid-liquid flow; Droplet deformation; Microfluidics.

$\begin{array}{ll}\text { Abbreviations } & \\ \text { CSF } & \text { Continuum Surface Force } \\ \text { DPD } & \text { Dissipative Particle Dynamics } \\ \text { LBM } & \text { Lattice Boltzmann Method } \\ \text { LGA } & \text { Lattice Gas Automata } \\ \text { LJ } & \text { Lennard-Jones } \\ \text { PPE } & \text { Pressure Poisson Equation } \\ \text { SPH } & \text { Smoothed Particle Hydrodynamics } \\ \text { VOF } & \text { Volume of Fluid }\end{array}$

\title{
1 Introduction
}

Processing of immiscible liquid-liquid dispersions occurs widely in the manufacture of foods [1], pharmaceuticals [2], cosmetics [3], paints [4], and oil [5]. Examples of processes include emulsification $[4,6]$, encapsulation $[7,8]$, multiphase reaction systems $[9,10]$, electrochemical 
processes [11], bioprocesses [12], separation processes [13] such as liquid-liquid extraction [14], polymer blending [15], and oil recovery [16]. Processes involving immiscible liquids are also found beyond industry, including in environmental clean-up [17], artificial oxygen carriers [18], oil spills [19], and pyroclastic flows [20].

There is an increasing need to understand and exploit the link between liquid-liquid phase morphology and fluid dynamics within the phases on the one hand and the conditions that lead to them on the other. For example, the droplet size distribution of an emulsion produced through agitation is a function of the balance between droplet breakup and coalescence, which can be controlled by the surfactant and stabilizer concentration and relative velocities of the phases [2123]. Microfluidic production of encapsulates provides a further example: in this case, encapsulate morphology can be varied through the nature of the flow-focusing in the microchannels and, amongst other things, the continuous-to-dispersed phase flow rate ratio [24, 25]. The challenges faced in experimentally elucidating these types of relationships are significant, however. For example, the visualization of the morphologies of the phases and flow fields therein are still very much in their infancy [26-29]. Models that treat the phases and interfaces between them explicitly have, therefore, a key role to play in building understanding of liquid-liquid systems and exploiting this understanding in a systematic way.

Models of liquid-liquid systems in which the individual phases and interfaces between them are treated explicitly are long-standing [30-33]. The earliest models, which focus on droplets in a continuous phase, include those of Taylor [34, 35], Mason and co-workers [36, 37], Cox and coworkers [38, 39], and Acrivos and co-workers [40-42] amongst others. Whilst these models were important in building understanding, they are limited by a good number of simplifying assumptions [43], including negligible inertial effects (i.e. small Reynolds numbers), small viscosity ratio ranges, and regular droplet shapes (e.g. spheres; ellipsoids). Phenomenological approaches have been adopted to overcome some limitations associated with wholly analytical models. For example, Maffettone and Minale [44] used such an approach, determining the model parameters by ensuring it matches analytical results in appropriate limits. Such models are, however, also limited by underpinning assumptions such as, for example, specific droplet shapes and the absence of any connection between the model parameters and underlying fundamentals.

Adoption of wholly numerical approaches can, in principle, overcome limitations faced by analytical and related models. Some of the earliest numerical models include those of Acrivos and co-workers $[43,45,46]$, who studied the deformation and rupture of a viscous droplet suspended in another liquid within extensional and shear flow fields. More recent examples include the works of Loewenberg, Hinch and Davis [47-53], which are based on a boundary-integral approach. Interface tracking techniques such as Volume of Fluid methods (VOF) [54, 55] and level set methods [56, 57] have been used even more recently. Whilst these works represent major advances in the field, 
they have two significant disadvantages that lead to algorithmic complexity, computational expense and numerical stability challenges [33, 58]: (1) the need to adjust the mesh as the phases deform; and (2) the need to track the interfaces between the different phases. Additionally, the treatment of coalescence and rupture of the interfaces between the phases is extremely challenging if not impossible in many of these techniques $[59,60]$.

Particle-based approaches have more recently been used as a remedy to the issues faced by mesh-based approaches. The most widely-adopted is the Lattice Boltzmann method (LBM) [61, 62]. This method as well as its less widely-used antecedent, Lattice-Gas Automata (LGA) [63, 64], are also not without their problems and limitations, however. For example, Galilean invariance is lost in LGA, leading to a spurious density-dependent factor multiplying the inertial term in the momentum conservation equation whose effect cannot be removed [65]. LBM-based simulation of immiscible multiphase fluid flows, on the other hand, tend to suffer from stability problems [66]. An alternative approach is Dissipative Particle Dynamics (DPD) [58, 65, 67], which has been used to study the rheology of complex fluids [68]. A related but computationally far cheaper approach [69] of much longer standing is Smoothed Particle Hydrodynamics (SPH) [70]. In addition to avoiding all the issues associated with mesh-based methods, SPH has the advantages that it is derived directly from the underlying equations of continuum mechanics and, hence, can be easily extended to any non-Newtonian matter.

In addition to being used to model multiphase fluid systems via the two-fluid model [71, 72], SPH has been used extensively to model such systems in which the interfaces are explicitly resolved. Many of these ignore surface tension (e.g. [73-78]), making them of limited value when interest lies in liquid-liquid systems where it plays a key role in dictating behaviour. Some have, however, sought to include surface tension effects. The first broad approach is that initially proposed by Morris [79], which embeds the continuum surface force (CSF) approach [80] within SPH. This approach is disadvantaged by the need to evaluate the curvature of interfaces, a computationally challenging task that also leads to numerical instabilities. Adams and co-workers [81] addressed this issue, although computational complexity and expense is still an issue. The CSF-based SPH approach has since been extended to systems involving solid surfaces of desired wettability [81, 82] and strictly incompressible systems [83-90]. A second broad approach sees SPH combined with the Cahn-Hilliard model [91, 92]; this diffuse interface approach is yet to see wider use, however. The final broad approach to including surface tension within SPH is via incorporation of inter-particle forces that lead to phase separation. The earliest example of this approach was due to Nugent and Posch [93], whose inter-particle force was inspired by the intermolecular interaction term that arises in the van der Waals equation of state used to close their compressible SPH model. This particular model, which is far simpler and cheaper than the two alternative approaches mentioned above, has seen some use since (e.g. [94, 95]) along with similar models based on other inter-particle force models, including some inspired by DPD $[96,97]$ along with various other 
more arbitrary forms [98], including trigonometric [99] and inverse square law [100]. In all these cases, however, strict incompressibility has not been imposed; this has only been done once previously [101] using the trigonometric interaction model.

In this paper, we report incorporation of surface tension within strictly incompressible SPH framework by including a Lennard-Jones (LJ) interaction between particles. The adoption of the LJ interaction to bring about surface tension has the advantage that it mirrors the almost universally used approach to incorporating solid boundaries within $\mathrm{SPH}$, thus opening the way to unifying the treatment of interfaces in SPH. The paper first provides details of the SPH model and how it is parametrized to yield the desired surface tension. The new method is then validated by comparing its predictions to analytical results and experiments for a number of model problems, including deformation of a liquid droplet in a linear shear field and the same for a freely falling droplet in a quiescent continuous phase.

\section{The Method}

\subsection{Governing Equations}

Smoothed-particle hydrodynamics builds on the Navier-Stokes equations expressed in the Lagrangian frame, which for an incompressible, isothermal system are of the form

$$
\begin{aligned}
& \nabla \cdot \mathbf{v}=0 \\
& \rho \frac{d \mathbf{v}}{d t}=\nabla \cdot \boldsymbol{\sigma}+\rho \mathbf{g}+\mathbf{F}_{I}
\end{aligned}
$$

where $\rho, \mathbf{v}$, and $\boldsymbol{\sigma}$ are the fluid density, velocity and stress tensor, respectively, and $\mathbf{g}$ is the acceleration due to body forces such as gravity. The term $\mathbf{F}_{I}$ is introduced into the momentum equation here to enable the imposition of immiscibility between the different liquids as described in more detail below. The form of this force is selected in a way that its sum across the entire multiphase system disappears, ensuring overall conservation of momentum.

The stress tensor is described here by

$$
\boldsymbol{\sigma}=-P \mathbf{I}+\boldsymbol{\tau}
$$

where $P$ is the pressure, $\mathbf{I}$ the unit tensor, and $\boldsymbol{\tau}$ the shear stress tensor. The latter for a Newtonian fluid may be expressed as

$$
\mathbf{\tau}=-\mu\left[\nabla \mathbf{v}+(\nabla \mathbf{v})^{T}\right]
$$

where $\mu$ is the dynamic viscosity. 


\subsection{SPH Discretization}

In $\mathrm{SPH}$, the fluid is represented by a discrete set of particles of mass, $m_{i}$, and viscosity, $\mu_{i}$, that move with the local fluid velocity, $\mathbf{v}_{i}$. Here, each particle also carries a colour, $c_{i}$, to indicate which of the immiscible fluids it belongs to. In principle, the approach here allows for any number of colours (i.e. fluids), each with its own viscosity and density - this opens the way for modelling systems with arbitrary sets of viscosity and density ratios.

The velocity and other quantities associated with any particle are interpolated at a position, $r$, through a weighted summation of contributions from all neighboring particles within a compact support of radius $O(h)$ as illustrated in Figure 1, where $h$ is selected to balance the need for the weighting function, $W(h)$, to sample a sufficient number of particles-j around particle-i whilst not being so great that it deviates significantly from the ideal [70]. For example, the density of a particle$i$ is given by [102]

$$
\rho_{i}=\sum_{j} m_{j} W\left(r_{i j}, h\right)
$$

where $r_{i j}$ is the distance between particles $i$ and $j$.

The pressure gradient associated with particle-i is given by $[102,103]$

$$
\nabla P_{i}=\rho_{i} \sum_{j} m_{j}\left(\frac{P_{j}}{\rho_{j}^{2}}+\frac{P_{i}}{\rho_{i}^{2}}\right) \nabla_{i} W_{i j}
$$

where $P_{i}$ is the pressure associated with particle- $i$.

Finally, the divergence of the shear stress tensor attached to a particle-i is given by [104]

$$
(\nabla . \boldsymbol{\tau})_{i}=\rho_{i} \sum_{j} m_{j}\left(\frac{\boldsymbol{\tau}_{j}}{\rho_{j}^{2}}+\frac{\boldsymbol{\tau}_{i}}{\rho_{i}^{2}}\right) \cdot \nabla_{i} W_{i j}
$$

The components of the shear stress tensor in this expression, which are derived from Equation (4), are given by [105]

$$
\tau_{i}^{\alpha \beta}=-\left(\sum_{j} \frac{m_{j} \mu_{j}}{\rho_{j}} v_{i j}^{\beta} \frac{\partial W_{i j}}{\partial x_{i}^{\alpha}}+\sum_{j} \frac{m_{j} \mu_{j}}{\rho_{j}} v_{i j}^{\alpha} \frac{\partial W_{i j}}{\partial x_{i}^{\beta}}\right)
$$

where $\mathbf{v}_{i j}=\mathbf{v}_{i}-\mathbf{v}_{j}$.

We have employed the following quintic spline kernel to ensure the second derivatives of the smoothing kernel that arise in the viscous SPH model are smooth, thus avoiding instabilities that are known to arise with lower order kernels $[106,107]$ 


$$
W\left(r^{*}, h\right)=\frac{7}{478 \pi h^{2}} \times \begin{cases}\left(3-r^{*}\right)^{5}-6\left(2-r^{*}\right)^{5}+15\left(1-r^{*}\right)^{5} & 0 \leq r^{*}<1 \\ \left(3-r^{*}\right)^{5}-6\left(2-r^{*}\right)^{5} & 1 \leq r^{*}<2 \\ \left(3-r^{*}\right)^{5} & 2 \leq r^{*}<3 \\ 0 & r^{*}>3\end{cases}
$$

where $r^{*}=r / h$ is a dimensionless radius.

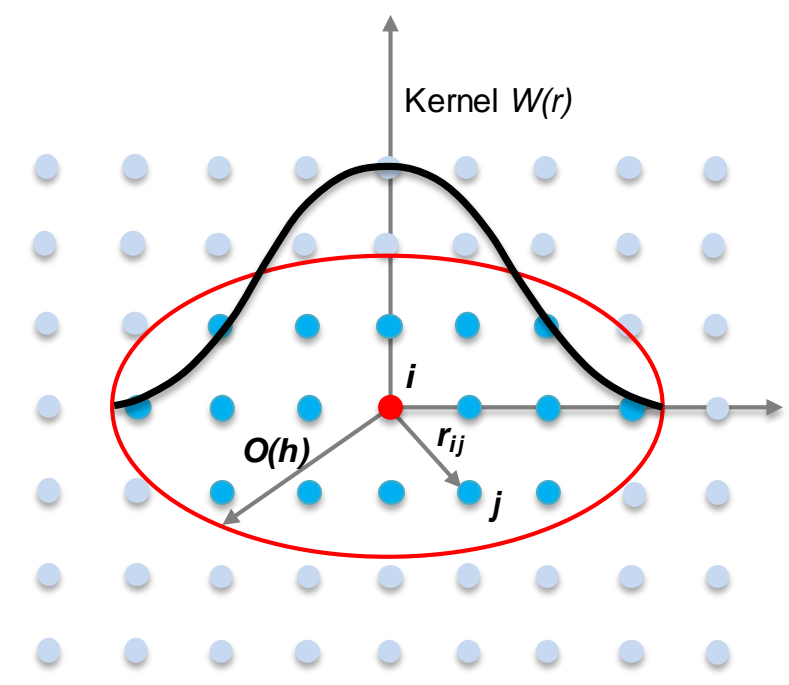

Fig. 1 An illustration of an SPH weighting function (solid black line) with compact support of radius $\mathrm{O}(h)$ (closed red arc) sited on particle- $i$ (shown in red) that leads to the particle interacting with all other particles- $j$ within the support radius (shown in blue).

\subsection{Immiscibility Model}

The immiscibility between the different fluids is enforced through the force, $F_{I}$, in Equation (2), which is dependent on the colour of the particles. We have adopted a force that is based on the following functional form that is motivated by the Lennard-Jones ( $\mathrm{LJ}$ ) interaction model

$$
\phi_{i j}= \begin{cases}-\varepsilon_{i j}\left(\frac{L_{0}}{r}\right)^{6} & \text { if } c_{i}=c_{j} \\ \varepsilon_{i j}\left(\frac{L_{0}}{r}\right)^{12} & \text { if } c_{i} \neq c_{j}\end{cases}
$$

where $L_{0}$ is a reference length and $\varepsilon_{i j}$ dictates the surface tension for the fluid pair as outlined further in sub-section 3.1 below (c.f. Figure 3 and associated text). The first part of this expression, which yields an attractive force between particles of the same colour (i.e. same fluid), leads to clustering of like-colored particles and is, therefore, the primary driving force for phase separation in the model. Restricting this term to like-colored particles is akin to setting the ratio $\varepsilon_{i j} / \varepsilon_{i i}=0$ for $i \neq j$, which has been shown to yield the sharpest interface between the phases in molecular simulations [108]. Experimentation by us supported this notion. The second term further limits intermixing of different fluids by inducing a very short range repulsive force between particles of different 
colour. Although the 12-6 form of the LJ model is widely used in $\mathrm{SPH}$, out of interest we did experiment with other exponent pairs (e.g. 12-12, 12-4) but found none yielded behaviour superior to that given by the more commonly used form.

The force between SPH particles that induces immiscibility is obtained from the gradient of the LJ expression

$$
\mathbf{F}_{I}=-\nabla \phi
$$

In SPH form, this equation leads to the following force acting on particle-i due to surface tension

$$
\left(\mathbf{F}_{I}\right)_{i}=\sum_{j} \frac{m_{j}}{\rho_{j}} \phi_{i j} \nabla_{i} W_{i j}
$$

The motivation for adopting the 12-6 LJ model here is two-fold. It has long been known that this model admits phase separation in the molecular context when the kinetic energy of the particles (which is quantified by the temperature) is smaller than a critical value of $\varepsilon_{i j}[108,109]$. It is also widely used in SPH to include solid surfaces and, as such, its use here opens the pathway to using a unified approach to including solid/fluid and fluid/fluid interfaces with desired wettabilities and surface tensions, respectively.

\subsection{Solution Algorithm for Incompressible Flow of Immiscible Fluids}

Combining Equations (2), (6), (7), and (12) leads to the following SPH formulation for the momentum equation

$$
\frac{\mathrm{d} \mathbf{v}_{i}}{d t}=-\sum_{j} m_{j}\left(\frac{P_{j}}{\rho_{j}^{2}}+\frac{P_{i}}{\rho_{i}^{2}}\right) \nabla_{i} W_{i j}+\sum_{j} m_{j}\left(\frac{\mathbf{\tau}_{j}}{\rho_{j}^{2}}+\frac{\boldsymbol{\tau}_{i}}{\rho_{i}^{2}}\right) \cdot \nabla_{i} W_{i j}+\mathbf{g}-\frac{1}{\rho_{i}} \sum_{j} \frac{m_{j}}{\rho_{j}} \phi_{i j} \nabla_{i} W_{i j}
$$

This is solved using a two-step predictor-corrector scheme based on that proposed by Cummins and Rudman [110] for single phase strictly incompressible flows and later extended to multiphase flows without surface tension by Shao and Lo [111]. Firstly, an initial estimate of the particle velocities at time $t+\Delta t, \tilde{\mathbf{v}}$, is made using only the shear stress, $\mathbf{F}_{\mathbf{\tau}}$, body, $\mathbf{F}_{\mathbf{g}}$, and surface force, $\mathbf{F}_{\boldsymbol{\phi}}$, terms (i.e. last three terms) in Equation (13) at time step-t (indicated by the subscript- $t$ ) [110, 111].

$$
\tilde{\mathbf{v}}=\mathbf{v}_{t}+\left(\mathbf{F}_{\mathbf{\tau}}+\mathbf{F}_{\mathbf{g}}+\mathbf{F}_{\boldsymbol{\phi}}\right)_{t} \Delta t
$$

The corresponding initial estimates for the particle positions are then evaluated using [111]

$$
\tilde{\mathbf{x}}=\mathbf{x}_{t}+\tilde{\mathbf{v}} \Delta t
$$

At this point incompressibility has not been enforced, and thus the density obtained from use of these initial particle position estimates in Equation (5), $\tilde{\rho}$, will be unlikely to be equal to that desired. 
The desired density is recovered by correcting the initial estimates of the particle velocities using [111]

$$
\mathbf{v}_{t+1}=\widetilde{\mathbf{v}}+\Delta \widetilde{\mathbf{v}}
$$

where the velocity correction is evaluated using the pressure gradient term of the momentum equation only

$$
\Delta \tilde{\mathbf{v}}=-\frac{1}{\tilde{\rho}} \nabla P_{t+1} \Delta t
$$

To obtain the pressure at the new time, $P_{t+1}$, Equations (16) and (17) are combined and the divergence is taken to give [110]

$$
\nabla \cdot\left(\frac{\mathbf{v}_{t+1}-\tilde{\mathbf{v}}}{\Delta \mathrm{t}}\right)=-\nabla \cdot\left(\frac{1}{\tilde{\rho}} \nabla P_{t+1}\right)
$$

Imposing the incompressibility condition at the new time step, $\nabla \cdot \mathbf{v}_{\mathrm{t}+1}=0$, leads to the Pressure Poisson Equation (PPE) [110]

$$
\nabla \cdot\left(\frac{1}{\tilde{\rho}} \nabla P_{t+1}\right)=\frac{\nabla \cdot \tilde{\mathbf{v}}}{\Delta \mathrm{t}}
$$

The left hand side is discretized based on Shao's approximation for the Laplacian in SPH [111], which is a hybrid of a standard SPH first derivative with a finite difference computation

$\nabla \cdot\left(\frac{1}{\rho} \nabla P\right)_{i}=\sum_{j} m_{j} \frac{8}{\left(\rho_{i}+\rho_{j}\right)^{2}} \frac{\left(P_{i}-P_{j}\right) \mathbf{r}_{\mathrm{ij}} \cdot \nabla_{i} W_{i j}}{\left|\mathbf{r}_{i j}\right|^{2}+\omega^{2}}$

where $\omega$ is a small value (e.g. $0.1 h$ ) to ensure the denominator is always non-zero.

The right hand side of equation (19) is discretized in SPH using [110]

$$
(\nabla . \widetilde{\mathbf{v}})_{i}=\rho_{i} \sum_{j} m_{j}\left(\frac{\widetilde{\mathbf{v}}_{j}}{\rho_{j}^{2}}+\frac{\widetilde{\mathbf{v}}_{i}}{\rho_{i}^{2}}\right) \cdot \nabla_{i} W_{i j}
$$

Discretization of the PPE equation leads to a system of linear equations, $\mathbf{A y}=\mathbf{b}$, in which $\mathbf{y}$ is the vector of unknown pressure gradients to be determined, and the matrix $\mathbf{A}$ is not necessarily positive definite or symmetric. In the present work, the biconjugate gradient algorithm [112] was used to solve this set of equations.

Once the velocity at the next time step is determined via use of Equation (16), the new particle positions are finally obtained using

$$
\mathbf{x}_{t+1}=\mathbf{x}_{t}+\frac{1}{2}\left(\mathbf{v}_{t}+\mathbf{v}_{t+1}\right) \Delta t
$$




\section{Results and Discussion}

\subsection{Young-Laplace and Parameterization of Immiscibility Model}

The validity of the new method was first assessed by ensuring that an initially square droplet transformed into a circular droplet that conforms to the Young-Laplace equation. The two liquids were quiescent and of the same density $\left(\eta=\rho_{d} / \rho_{c}=1\right)$ and viscosity $\left(\lambda=\mu_{d} / \mu_{c}=1\right)$. The droplet was initially a square of size $6 h \times 6 h\left(h=1.25 L_{0}\right.$, where $\left.L_{0}=5 \times 10^{-5} \mathrm{~m}\right)$ within a periodic domain of $24 h \times 24 h$; the total number of SPH particles was $N_{p}=961$. A timestep size of $\Delta t^{*}=$ $\Delta t \sqrt{\gamma / \rho h^{3}}=0.1$ was used.

Figure 2 shows, as expected, that the perimeter of the droplet, $C_{d}$, gradually decreases as it changes from its initial square shape to a circle. This figure also shows that the pressure difference across the interface between the two phases, shown in dimensionless form, $\Delta P^{*}=\Delta P h / \gamma$, increases as the droplet transforms in shape, in line with the physics where the pressure difference is counter-balanced by the surface tension (in this case, $\gamma=0.045 \mathrm{~N} / \mathrm{m}$, which corresponds to $\varepsilon=$ $\left.56.25 \mathrm{~N} / \mathrm{m}^{2}\right)$.

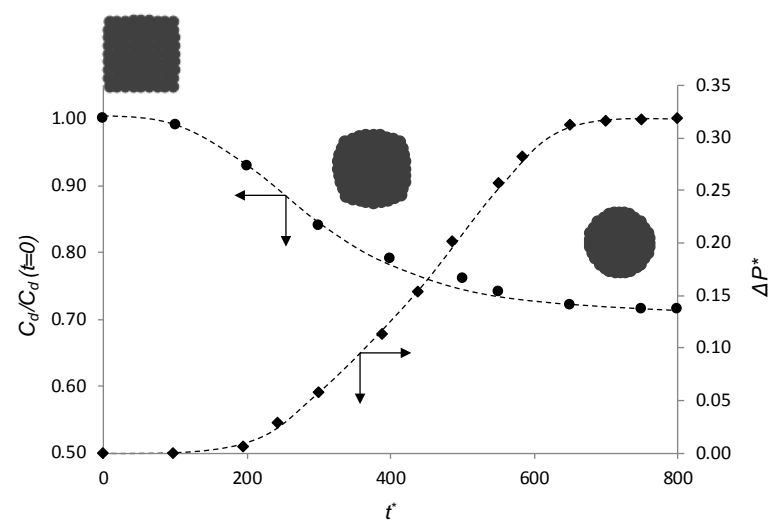

Fig. 2 Variation through time, $t^{*}=t \sqrt{\gamma / \rho h^{3}}$, of the circumference, $C_{d}$, of an initially square, neutrally buoyant, stationary droplet suspended in a second continuous phase and the associated change in pressure difference, $\Delta P^{*}=\Delta P h / \gamma$, across the interface between the two liquids. Snapshots of the droplet along the transformation pathway are shown at various points in the transformation.

Figure 3(a) shows the variation of the pressure droplet across the interface between the droplet and continuous phase obtained as a function of the inverse of the droplet size. The linear behaviour seen in this figure clearly conforms to the Young-Laplace equation

$$
\Delta P=\frac{\gamma}{R}
$$

This expression shows that the surface tension between two fluids can be straightforwardly equated to the gradient of the line in Figure 3(a). 

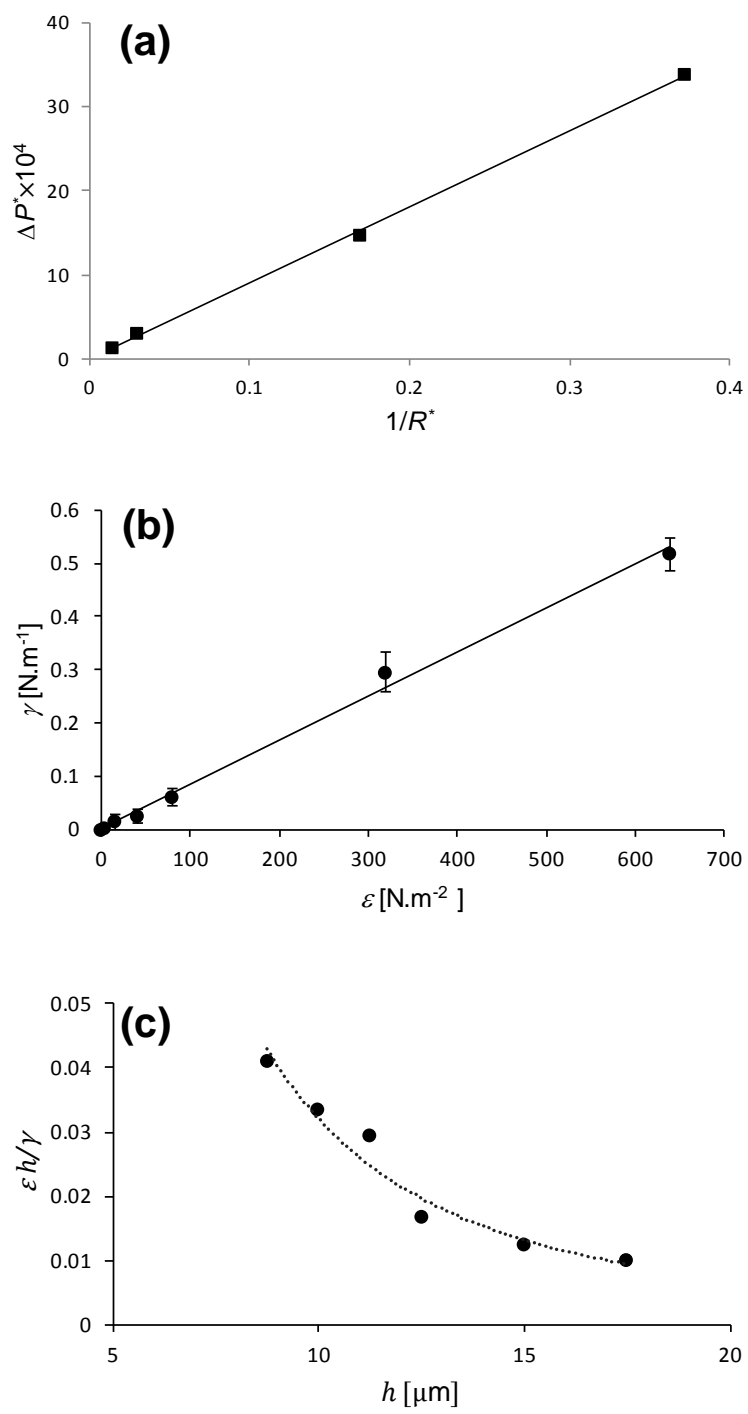

Fig. 3. Demonstration that the immiscibility model conforms to the Young-Laplace equation and use of this equation to determine the relationship between the immiscibility model parameter, $\varepsilon_{i j}$, and the surface tension, $\gamma_{i j}$, between two fluids of colour $c_{i}$ and $c_{j}$ : (a) variation of dimensionless pressure drop across the droplet interface with the inverse of its dimensionless radius, $R^{*}=R / h$, obtained using $\varepsilon=16 \mathrm{~N} / \mathrm{m}^{2}$ - the slope of this line is $\gamma=0.0142 \mathrm{~N} / \mathrm{m}$; (b) variation of the surface tension for a pair of fluids with the immiscibility model parameter obtained for a single SPH smoothing length ( $h=1.25 \times 10^{-5}$ in this case) - the error bars show relative error in the interfacial tension values evaluated from the time series obtained for each simulation; and (c) variation of the ratio of the immiscibility model parameter and interfacial tension with the SPH smoothing length.

The relationship between the immiscibility model parameter in Equation (10) for a fluid pair, $\varepsilon_{i j}$, and the interfacial tension, $\gamma_{i j}$, can be determined by repeating the simulations that lead to Figure 3(a) for various values of the former. Doing this leads to Figure 3(b), which shows that the interaction model parameter varies in a linear fashion with the interfacial tension. The slope of this linear relationship is dependent on the smoothing length as revealed in Figure 3(c). 


\subsection{Deformation of a Confined Droplet in a Linear Shear Field}

The new method was further validated by considering the deformation of an initially circular droplet under linear shear in a second immiscible liquid as illustrated in Figure S1; for simplicity, the densities and viscosities of the two liquids were the same (i.e. $\eta=\rho_{d} / \rho_{c}=1$ and $\lambda=\mu_{d} / \mu_{c}=1$ ). A droplet of diameter $D=17 h\left(h=1.2 L_{0}\right.$, where $\left.L_{0}=1 \times 10^{-5} \mathrm{~m}\right)$ was initially located centrally within a domain of height $H=34 h$ and length $L=100 h$; the total number of SPH particles was $N_{p}=4961$. To compensate truncation of the support domain of the kernel at the wall boundary, $3 h$ layers of uniformly spaced dummy particles were arranged on the outside of the boundary walls $[113,114]$ (see Figure S1). Equal but opposite velocities of magnitude $U$ were applied to the top and bottom of the domain with no slip boundary conditions (i.e. the top and bottom were treated as moving solid surfaces) to yield a linear shear field with shear rate $G=2 U / H$. The state of the system was evolved through time using timesteps of size $\Delta t^{*}=G \Delta t=0.001$.

Under suitable conditions, the initially stationary circular droplet deforms to take on a stable ellipsoidal shape as illustrated in Figure S1 and Figure S2, which illustrates that a sharp interface between the two liquids is obtained even when the original droplet deforms significantly. The character of this droplet may be described in part by the deformation parameter [115]

$$
D_{f}=\frac{a-b}{a+b}
$$

where $a$ and $b$ are the lengths of the major and minor axes of the ellipsoid, respectively. Figure 4 compares this parameter as obtained from the SPH simulations at a confinement ratio of $D / H=$ 0.5 with the experimental results of Sibillo et al. [116] and Taylor's model for an unconfined droplet in a shear field [115]

$$
D_{f}=\frac{19 \lambda+16}{16 \lambda+16} C a
$$

where $C a=\mu G D / \gamma$ is the Capillary number. This figure shows that the deformations predicted by SPH match very well the experimental results up to the $C a=0.3$ limit probed by Sibillo et al. [116], with little dependency on the number of SPH particles. The results also match well those predicted by Taylor's model up to this Capillary Number. However, beyond this one can see a significant deviation from Taylor's model, indicating that confinement has an effect at higher Capillary Numbers where shear is starting to dominate over surface tension. 


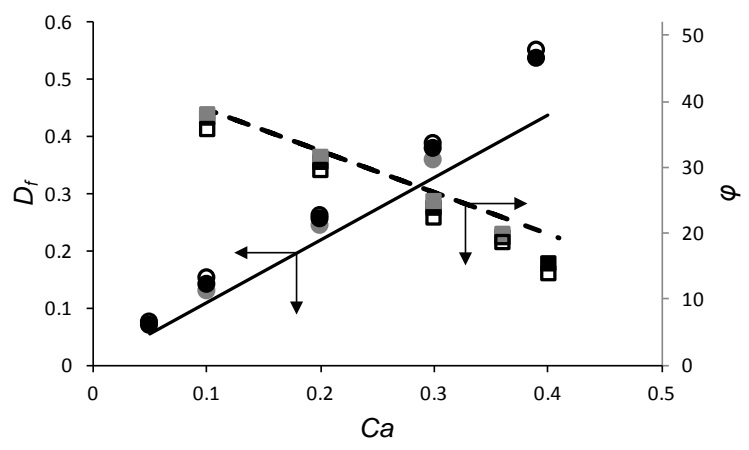

Fig. 4 Variation of the deformation parameter (circles and solid line) as defined in Equation (24) and droplet tilt angle as defined in Figure S1 (squares and broken line) with Capillary Number,

$C a=\mu G D / \gamma$, as predicted by SPH with $N_{p}=7701, L_{0}=8.0 \times 10^{-6}$ and $h=1.2 \times L_{0}$ (solid points), SPH with $N_{p}=4961, L_{0}=1.0 \times 10^{-5}$ and $h=1.2 \times L_{0}$ (open points), experimental data of Sibillo et al. [116] (shaded points), and Taylor theory [115] as defined by Equations (25) and (26) (lines).

The other key parameter characterizing the droplet behaviour is the angle the major axis of the ellipsoid subtends to the primary direction of flow, $\varphi$ (see Figure S1). Figure 4 also shows a comparison of this angle as obtained from the SPH simulations with the experimental data and Taylor's model [115]

$$
\varphi=\frac{\pi}{4}-\frac{(19 \lambda+16)(2 \lambda+3)}{80(\lambda+1)} C a
$$

Once again, comparison with experiment is very good. The deviation of the SPH values from Taylor's theory is modest but grows with Capillary number, indicating that the tilt angle is also sensitive to confinement in the same way as the deformation parameter is.

The model can be used to understand the behaviour of droplets in such systems as a function of the key dimensionless groups such as Capillary number. This is illustrated in Figure 5 where the SPH model is used to model droplet rupture (see Figure S3 and associated text for greater details). We will report at a later date the use of the SPH model to evaluate the critical Capillary Number for rupture as a function of the various physical parameters that characterize this system.
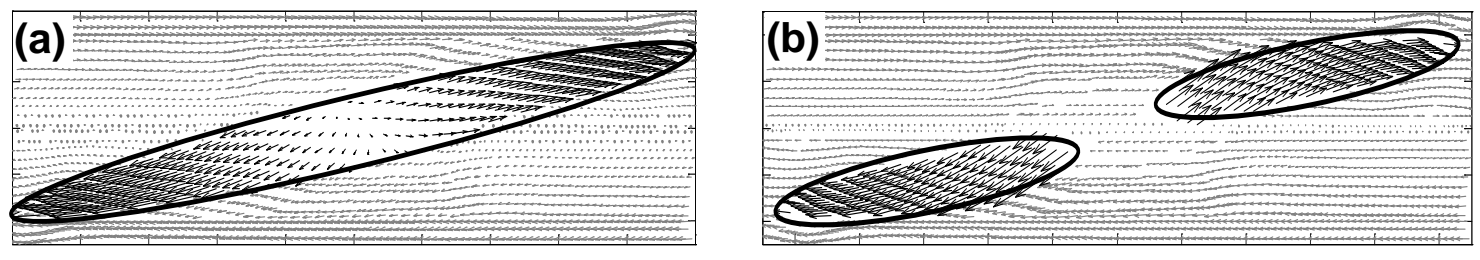

Fig. 5 The new model yields velocity fields in both phases and droplet morphology change without complex interface tracking algorithms, which is particularly important for stable simulation of droplet break-up as shown here for $C a=0.5, \operatorname{Re}=0.1, \lambda=1, D / H=0.5, N_{p}=4961, L_{0}=$ $1.0 \times 10^{-5}$, and $h=1.2 \times L_{0}$ : (a) prior to droplet rupture; and (b) after droplet rupture as the two daughter drops move away from each other. 


\subsection{Deformation of Free-Falling Droplet}

The new method was finally validated by considering the descent of an initially stationary circular droplet accelerating under gravity through a second stationary liquid whose density, $\rho_{d}=$ $1150 \mathrm{~kg} / \mathrm{m}^{3}$ differed from that of the continuous phase, $\rho_{c}$, by $\Delta \rho=\rho_{d}-\rho_{c}=150 \mathrm{~kg} / \mathrm{m}^{3}$. The droplet was initially of diameter $D=14 h\left(h=1.2 L_{0}\right.$, where $\left.L_{0}=1 \times 10^{-4} \mathrm{~m}\right)$, and was located within a periodic domain of size $34 h \times 34 h$; the total number of SPH particles was $N_{p}=1681$. The droplet motion and deformation was followed through time $\left(\Delta t^{*}=\Delta t \sqrt{g / D}=0.004\right)$ until the terminal velocity was reached and the droplet shape remained unchanged $\left(t^{*}=45\right)$. This problem is characterized by four dimensionless numbers: the Morton Number, Mo $=g \Delta \rho \mu_{c}^{4} / \rho_{c}^{2} \gamma^{3}$, Ohnesorge Numbers of the dispersed and continuous phase, $\mathrm{Oh}_{d}=\mu_{d} / \sqrt{\rho_{d} \gamma D}$, and $\mathrm{Oh}_{c}=$ $\mu_{c} / \sqrt{\rho_{c} \gamma D}$ respectively, and Eötvös Number, Eo $=g \Delta \rho D^{2} / \gamma$.

Figure 6 compares the variation with time of the droplet descent speed, $u_{d}^{*}=u_{d} / \sqrt{g D}$, obtained here from SPH with that predicted by Han and Tryggvason [117] using a finite difference front tracking method. This figure shows that the two predictions are very similar, with an average difference of about 1.4\%. Snapshots of the deforming droplet at various times indicate the coupling between the deformation and droplet descent speed. After the initial rapid rise in the speed of the droplet, it first decelerates as its tail deforms before mildly accelerating again to steady state.

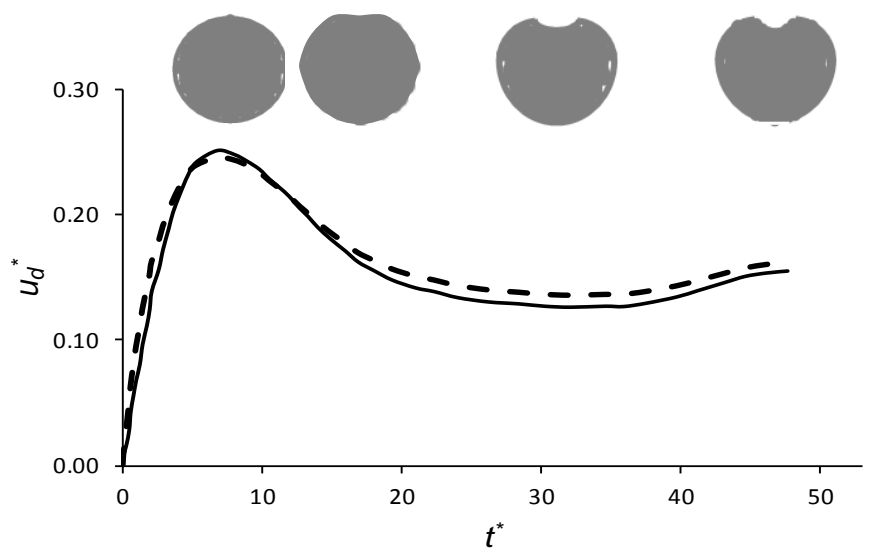

Fig. 6 Variation of the droplet descent speed with time as predicted by SPH (broken line) and Han and Tryggvason [117] (solid line) for $\eta=1.15, \lambda=1$, Eo $=10, \mathrm{Oh}_{d}=0.24$ and $\mathrm{Mo}=0.04$. Snapshots of the deforming droplet are shown as inserts at various times.

As with droplets under shear, the SPH model provides the possibility of exploring the behaviour of falling drop systems as a function of the relevant system variables. This is illustrated in Figure S4, which show the results for droplet descent at two markedly different Eötvös Numbers, Eo. 


\section{Conclusion}

A new SPH-based approach was developed for incompressible immiscible liquid-liquid systems in which interfacial tension is important. The approach has a number of advantages over prior SPH models. It does not require any interface tracking or gradient evaluation at the interface, unlike the most widely used method in the field to date [79, 81-90]. Instead, phase separation with finite interfacial tension emerges naturally through the inclusion of a Lennard-Jones (LJ) interaction between particle pairs that is dependent upon the fluid species of the particles. The adoption of the LJ form here opens the way to unifying the treatment of all interfaces - fluid/solid, fluid/fluid and solid/solid - and associated interfacial energies. The method presented here can be straightforwardly extended to more than two fluids and allows for non-unity viscosity and density ratios. The SPH basis of the method means it can also be straightforwardly extended to nonNewtonian constitutive models.

The new approach was validated against different model problems including Young-Laplace equation, confined droplet deformation under a linear shear field, and a liquid droplet falling under gravity through another quiescent liquid. These results show that the model can predict the fluid dynamics of immiscible liquid-liquid systems in terms of both the phase morphologies and dynamics with no complex interface tracking algorithm or numerical stability issues that are commonly encountered with existing techniques $[54,55,57,61]$.

The use of the new approach to elucidate the behaviour of non-trivial immiscible liquid systems was also demonstrated. Of particular note is its use to model droplet break-up under shear, which can be straightforwardly modelled, unlike in many other approaches where the need to evaluate gradients at the interfaces between fluids makes interface rapture a challenge to capture if not impossible $[59,60]$. Work is now underway to use the new method to improve understanding of the link between phase morphology and intraphase flows of immiscible liquid flows in microfluidic devices and various system parameters such as viscosity ratios, Capillary number, hydrodynamic focusing micro-geometry and associated flowrate ratios, and wall wettabilities. This work is of particular relevance to microfluidic production of drug and vaccine formulations $[118,119]$ and multiphase microreactors $[120,121]$.

\section{Acknowledgments}

Access to the HPC systems of eResearch SA are acknowledged. Both HE and MNK also acknowledge the University of Adelaide for their IPRS, APA and ASI PhD scholarships. 


\section{References}

[1] H.D. Silva, M.Â. Cerqueira, A.A. Vicente, Nanoemulsions for food applications: development and characterization, Food and Bioprocess Technology 5 (2012) 854-867.

[2] F. Nielloud, Pharmaceutical emulsions and suspensions: revised and expanded, CRC Press 2000.

[3] R. Müller, R. Petersen, A. Hommoss, J. Pardeike, Nanostructured lipid carriers (NLC) in cosmetic dermal products, Advanced Drug Delivery Reviews 59 (2007) 522-530.

[4] J. Bibette, F. Leal-Calderon, V. Schmitt, P. Poulin, Emulsion science: Basic principles. An overview, Springer 2003.

[5] J. Sjöblom, N. Aske, I.H. Auflem, Ø. Brandal, T.E. Havre, Ø. Sæther, A. Westvik, E.E. Johnsen, H. Kallevik, Our current understanding of water-in-crude oil emulsions.: Recent characterization techniques and high pressure performance, Advances in Colloid and Interface Science 100 (2003) 399-473.

[6] E. Windhab, M. Dressler, K. Feigl, P. Fischer, D. Megias-Alguacil, Emulsion processing-from single-drop deformation to design of complex processes and products, Chemical Engineering Science 60 (2005) 2101-2113.

[7] S. Freitas, H.P. Merkle, B. Gander, Microencapsulation by solvent extraction/evaporation: reviewing the state of the art of microsphere preparation process technology, Journal of Controlled Release 102 (2005) 313-332.

[8] S. Köster, F.E. Angile, H. Duan, J.J. Agresti, A. Wintner, C. Schmitz, A.C. Rowat, C.A. Merten, D. Pisignano, A.D. Griffiths, Drop-based microfluidic devices for encapsulation of single cells, Lab on a Chip 8 (2008) 1110-1115.

[9] J.M. Asua, Emulsion polymerization: from fundamental mechanisms to process developments, Journal of Polymer Science Part A: Polymer Chemistry 42 (2004) 1025-1041.

[10] A. Campanella, M.A. Baltanás, Degradation of the oxirane ring of epoxidized vegetable oils in liquid-liquid heterogeneous reaction systems, Chemical Engineering Journal 118 (2006) 141-152.

[11] Z. Samec, Electrochemistry at the interface between two immiscible electrolyte solutions (IUPAC Technical Report), Pure and Applied Chemistry 76 (2004) 2147-2180.

[12] N. Reis, C. Goncalves, M. Aguedo, N. Gomes, J. Teixeira, A. Vicente, Application of a novel oscillatory flow micro-bioreactor to the production of $y$ decalactone in a two immiscible liquid phase medium, Biotechnology Letters 28 (2006) 485-490.

[13] C. Geankoplis, Transport processes and separation process principles, Prentice Hall Press2003. 
[14] S. Pedersen-Bjergaard, K.E. Rasmussen, Liquid-phase microextraction with porous hollow fibers, a miniaturized and highly flexible format for liquid-liquid extraction, Journal of Chromatography A 1184 (2008) 132-142.

[15] X. Zhang, Z. Wang, X. Dong, D. Wang, C.C. Han, Interplay between two phase transitions: Crystallization and liquid-liquid phase separation in a polyolefin blend, The Journal of Chemical Physics 125 (2006) 024907.

[16] S. Thomas, Enhanced oil recovery-an overview, Oil \& Gas Science and Technology-Revue de l'IFP 63 (2008) 9-19.

[17] C. Basheer, R. Balasubramanian, H.K. Lee, Determination of organic micropollutants in rainwater using hollow fiber membrane/liquid-phase microextraction combined with gas chromatography-mass spectrometry, Journal of Chromatography A 1016 (2003) 11-20.

[18] D.R. Spahn, Current status of artificial oxygen carriers, Advanced Drug Delivery Reviews 40 (2000) 143-151.

[19] P.S. Daling, M.Ø. Moldestad, Ø. Johansen, A. Lewis, J. Rødal, Norwegian testing of emulsion properties at sea-the importance of oil type and release conditions, Spill Science \& Technology Bulletin 8 (2003) 123-136.

[20] G. Chazot, H. Bertrand, J. Mergoil, S.M. Sheppard, Mingling of immiscible dolomite carbonatite and trachyte in tuffs from the Massif Central, France, Journal of Petrology 44 (2003) 1917-1936.

[21] P.J. Dowding, B. Vincent, Suspension polymerisation to form polymer beads, Colloids and Surfaces A: Physicochemical and Engineering Aspects 161 (2000) 259-269.

[22] X. Ni, D. Mignard, B. Saye, J. Johnstone, N. Pereira, On the evaluation of droplet breakage and coalescence rates in an oscillatory baffled reactor, Chemical Engineering Science 57 (2002) 2101-2114.

[23] V. Alopaeus, J. Koskinen, K.I. Keskinen, Simulation of the population balances for liquid-liquid systems in a nonideal stirred tank. Part 1 description and qualitative validation of the model, Chemical Engineering Science 54 (1999) 58875899.

[24] R.K. Shah, H.C. Shum, A.C. Rowat, D. Lee, J.J. Agresti, A.S. Utada, L.-Y. Chu, J.-W. Kim, A. Fernandez-Nieves, C.J. Martinez, Designer emulsions using microfluidics, Materials Today 11 (2008) 18-27.

[25] J. Wan, Microfluidic-based synthesis of hydrogel particles for cell microencapsulation and cell-based drug delivery, Polymers 4 (2012) 1084-1108.

[26] L. Liu, O.K. Matar, E.S.P. de Ortiz, G.F. Hewitt, Experimental investigation of phase inversion in a stirred vessel using LIF, Chemical Engineering Science 60 (2005) 85-94.

[27] L. Liu, O.K. Matar, C.J. Lawrence, G.F. Hewitt, Laser-induced fluorescence (LIF) studies of liquid-liquid flows. Part I: flow structures and phase inversion, Chemical Engineering Science 61 (2006) 4007-4021. 
[28] G. Seevaratnam, H. Ding, O. Michel, J. Heng, O. Matar, Laminar flow deformation of a droplet adhering to a wall in a channel, Chemical Engineering Science 65 (2010) 4523-4534.

[29] N.M. Hasan, B.J. Azzopardi, Imaging stratifying liquid-liquid flow by capacitance tomography, Flow Measurement and Instrumentation 18 (2007) 241246.

[30] J. Rallison, The deformation of small viscous drops and bubbles in shear flows, Annual Review of Fluid Mechanics 16 (1984) 45-66.

[31] H.A. Stone, Dynamics of drop deformation and breakup in viscous fluids, Annual Review of Fluid Mechanics 26 (1994) 65-102.

[32] V. Cristini, Y.-C. Tan, Theory and numerical simulation of droplet dynamics in complex flows-a review, Lab on a Chip 4 (2004) 257-264.

[33] R. Scardovelli, S. Zaleski, Direct numerical simulation of free-surface and interfacial flow, Annual Review of Fluid Mechanics 31 (1999) 567-603.

[34] G.I. Taylor, The viscosity of a fluid containing small drops of another fluid, Proceedings of the Royal Society of London. Series A, Containing Papers of a Mathematical and Physical Character (1932) 41-48.

[35] G. Taylor, The formation of emulsions in definable fields of flow, Proceedings of the Royal Society of London. Series A, Containing Papers of a Mathematical and Physical Character (1934) 501-523.

[36] F.-D. Rumscheidt, S. Mason, Particle motions in sheared suspensions XII. Deformation and burst of fluid drops in shear and hyperbolic flow, Journal of Colloid Science 16 (1961) 238-261.

[37] W. Bartok, S. Mason, Particle motions in sheared suspensions: VII. Internal circulation in fluid droplets (theoretical), Journal of Colloid Science 13 (1958) 293307.

[38] R. Cox, The deformation of a drop in a general time-dependent fluid flow, Journal of Fluid Mechanics 37 (1969) 601-623.

[39] S. Torza, R. Cox, S. Mason, Particle motions in sheared suspensions XXVII. Transient and steady deformation and burst of liquid drops, Journal of Colloid and Interface Science 38 (1972) 395-411.

[40] D. Barthes-Biesel, A. Acrivos, Deformation and burst of a liquid droplet freely suspended in a linear shear field, Journal of Fluid Mechanics 61 (1973) 1-22.

[41] A. Acrivos, T. Lo, Deformation and breakup of a single slender drop in an extensional flow, Journal of Fluid Mechanics 86 (1978) 641-672.

[42] T. Taylor, A. Acrivos, On the deformation and drag of a falling viscous drop at low Reynolds number, Journal of Fluid Mechanics 18 (1964) 466-476.

[43] J. Rallison, A. Acrivos, A numerical study of the deformation and burst of a viscous drop in an extensional flow, Journal of Fluid Mechanics 89 (1978) 191200. 
[44] P. Maffettone, M. Minale, Equation of change for ellipsoidal drops in viscous flow, Journal of Non-Newtonian Fluid Mechanics 78 (1998) 227-241.

[45] M. Kojima, E. Hinch, A. Acrivos, The formation and expansion of a toroidal drop moving in a viscous fluid, Physics of Fluids 27 (1984) 19-32.

[46] E. Hinch, A. Acrivos, Long slender drops in a simple shear flow, Journal of Fluid Mechanics 98 (1980) 305-328.

[47] M. Loewenberg, E. Hinch, Numerical simulation of a concentrated emulsion in shear flow, Journal of Fluid Mechanics 321 (1996) 395-419.

[48] M. Loewenberg, E. Hinch, Collision of two deformable drops in shear flow, Journal of Fluid Mechanics 338 (1997) 299-315.

[49] A.Z. Zinchenko, R.H. Davis, An efficient algorithm for hydrodynamical interaction of many deformable drops, Journal of Computational Physics 157 (2000) 539-587.

[50] A.Z. Zinchenko, R.H. Davis, Shear flow of highly concentrated emulsions of deformable drops by numerical simulations, Journal of Fluid Mechanics 455 (2002) 21-61.

[51] M. Loewenberg, Numerical simulation of concentrated emulsion flows, Journal of Fluids Engineering 120 (1998) 824-832.

[52] V. Cristini, J. Bławzdziewicz, M. Loewenberg, Drop breakup in threedimensional viscous flows, Physics of Fluids 10 (1998) 1781-1783.

[53] V. Cristini, J. Bławzdziewicz, M. Loewenberg, An adaptive mesh algorithm for evolving surfaces: simulations of drop breakup and coalescence, Journal of Computational Physics 168 (2001) 445-463.

[54] J. Li, Y.Y. Renardy, M. Renardy, Numerical simulation of breakup of a viscous drop in simple shear flow through a volume-of-fluid method, Physics of Fluids 12 (2000) 269-282.

[55] Y.Y. Renardy, M. Renardy, V. Cristini, A new volume-of-fluid formulation for surfactants and simulations of drop deformation under shear at a low viscosity ratio, European Journal of Mechanics-B/Fluids 21 (2002) 49-59.

[56] E. Bertakis, S. Groß, J. Grande, O. Fortmeier, A. Reusken, A. Pfennig, Validated simulation of droplet sedimentation with finite-element and level-set methods, Chemical Engineering Science 65 (2010) 2037-2051.

[57] J.-J. Xu, Z. Li, J. Lowengrub, H. Zhao, A level-set method for interfacial flows with surfactant, Journal of Computational Physics 212 (2006) 590-616.

[58] P. Espanol, M. Revenga, Smoothed dissipative particle dynamics, Physical Review E 67 (2003) 026705.

[59] J. Liu, N.-T. Nguyen, Numerical simulation of droplet-based microfluidics, Micro and Nanosystems 2 (2010) 193-201. 
[60] M. Lappa, Coalescence and non-coalescence phenomena in multi-material problems and dispersed multiphase flows: Part 2, a critical review of CFD approaches, FDMP: Fluid Dynamics \& Materials Processing 1 (2005) 213-234.

[61] S. Chen, G.D. Doolen, Lattice Boltzmann method for fluid flows, Annual Review of Fluid Mechanics 30 (1998) 329-364.

[62] H. Bararnia, S. Seyyedi, D. Ganji, B. Khorshidi, Numerical investigation of the coalescence and breakup of falling multi-droplets, Colloids and Surfaces A: Physicochemical and Engineering Aspects 424 (2013) 40-51.

[63] J.F. Olson, D.H. Rothman, Three-dimensional immiscible lattice gas: application to sheared phase separation, Journal of Statistical Physics 81 (1995) 199-222.

[64] D.H. Rothman, J.M. Keller, Immiscible cellular-automaton fluids, Journal of Statistical Physics 52 (1988) 1119-1127.

[65] P.V. Coveney, K.E. Novik, Computer simulations of domain growth and phase separation in two-dimensional binary immiscible fluids using dissipative particle dynamics, Physical Review E 54 (1996) 5134.

[66] P. Lallemand, L.-S. Luo, Theory of the lattice Boltzmann method: Dispersion, dissipation, isotropy, Galilean invariance, and stability, Physical Review E 61 (2000) 6546.

[67] P. Hoogerbrugge, J. Koelman, Simulating microscopic hydrodynamic phenomena with dissipative particle dynamics, EPL (Europhysics Letters) 19 (1992) 155.

[68] P.B. Warren, Dissipative particle dynamics, Current Opinion in Colloid \& Interface Science 3 (1998) 620-624.

[69] N. Filipovic, M. Ivanovic, M. Kojic, A comparative numerical study between dissipative particle dynamics and smoothed particle hydrodynamics when applied to simple unsteady flows in microfluidics, Microfluidics and Nanofluidics 7 (2009) 227-235.

[70] J.J. Monaghan, Smoothed particle hydrodynamics, Reports on Progress in Physics 68 (2005) 1703.

[71] J. Monaghan, A. Kocharyan, SPH simulation of multi-phase flow, Computer Physics Communications 87 (1995) 225-235.

[72] J. Monaghan, Implicit SPH drag and dusty gas dynamics, Journal of Computational Physics 138 (1997) 801-820.

[73] J. Monaghan, A. Rafiee, A simple SPH algorithm for multi-fluid flow with high density ratios, International Journal for Numerical Methods in Fluids 71 (2013) 537561.

[74] M. Antuono, A. Colagrossi, D. Le Touzé, J. Monaghan, Conservation of circulation in SPH for 2D free-surface flows, International Journal for Numerical Methods in Fluids 72 (2013) 583-606. 
[75] J.J. Monaghan, Simulating free surface flows with SPH, Journal of Computational Physics 110 (1994) 399-406.

[76] B.M. Savage, M.C. Johnson, Flow over ogee spillway: Physical and numerical model case study, Journal of Hydraulic Engineering (2001).

[77] M. Prakash, P.W. Cleary, Three dimensional modelling of lava flow using Smoothed Particle Hydrodynamics, Applied Mathematical Modelling 35 (2011) 3021-3035.

[78] S. Cummins, T. Silvester, P.W. Cleary, Three-dimensional wave impact on a rigid structure using smoothed particle hydrodynamics, International journal for Numerical Methods in Fluids 68 (2012) 1471-1496.

[79] J.P. Morris, Simulating surface tension with smoothed particle hydrodynamics, International Journal for Numerical Methods in Fluids 33 (2000) 333-353.

[80] J. Brackbill, D.B. Kothe, C. Zemach, A continuum method for modeling surface tension, Journal of Computational Physics 100 (1992) 335-354.

[81] X. Hu, N.A. Adams, A multi-phase SPH method for macroscopic and mesoscopic flows, Journal of Computational Physics 213 (2006) 844-861.

[82] T. Breinlinger, P. Polfer, A. Hashibon, T. Kraft, Surface tension and wetting effects with smoothed particle hydrodynamics, Journal of Computational Physics 243 (2013) 14-27.

[83] X. Xu, J. Ouyang, T. Jiang, Q. Li, Numerical analysis of the impact of two droplets with a liquid film using an incompressible SPH method, Journal of Engineering Mathematics 85 (2014) 35-53.

[84] M. Tong, D.J. Browne, An incompressible multi-phase smoothed particle hydrodynamics $(\mathrm{SPH})$ method for modelling thermocapillary flow, International Journal of Heat and Mass Transfer 73 (2014) 284-292.

[85] M.S. Shadloo, M. Yildiz, Numerical modeling of Kelvin-Helmholtz instability using smoothed particle hydrodynamics, International Journal for Numerical Methods in Engineering 87 (2011) 988-1006.

[86] X. Hu, N.A. Adams, An incompressible multi-phase SPH method, Journal of Computational Physics 227 (2007) 264-278.

[87] X. Hu, N. Adams, A constant-density approach for incompressible multi-phase SPH, Journal of Computational Physics 228 (2009) 2082-2091.

[88] A. Zainali, N. Tofighi, M.S. Shadloo, M. Yildiz, Numerical investigation of Newtonian and non-Newtonian multiphase flows using ISPH method, Computer Methods in Applied Mechanics and Engineering 254 (2013) 99-113.

[89] M. Shadloo, A. Zainali, M. Yildiz, Simulation of single mode Rayleigh-Taylor instability by SPH method, Computational Mechanics 51 (2013) 699-715.

[90] N. Tofighi, M. Yildiz, Numerical simulation of single droplet dynamics in threephase flows using ISPH, Computers \& Mathematics with Applications 66 (2013) 525-536. 
[91] Z. Xu, P. Meakin, A.M. Tartakovsky, Diffuse-interface model for smoothed particle hydrodynamics, Physical Review E 79 (2009) 036702.

[92] A. Das, P. Das, Incorporation of diffuse interface in smoothed particle hydrodynamics: implementation of the scheme and case studies, International Journal for Numerical Methods in Fluids 67 (2011) 671-699.

[93] S. Nugent, H. Posch, Liquid drops and surface tension with smoothed particle applied mechanics, Physical Review E 62 (2000) 4968.

[94] Y. Melean, L.D.G. Sigalotti, A. Hasmy, On the SPH tensile instability in forming viscous liquid drops, Computer Physics Communications 157 (2004) 191-200.

[95] Y. Meleán, L.D.G. Sigalotti, Coalescence of colliding van der Waals liquid drops, International Journal of Heat and Mass Transfer 48 (2005) 4041-4061.

[96] C. Thieulot, L. Janssen, P. Español, Smoothed particle hydrodynamics model for phase separating fluid mixtures. I. General equations, Physical Review E 72 (2005) 016713.

[97] M. Liu, P. Meakin, H. Huang, Dissipative particle dynamics with attractive and repulsive particle-particle interactions, Physics of Fluids 18 (2006) 017101.

[98] W.G. Hoover, C.G. Hoover, Smooth-particle phase stability with generalized density-dependent potentials, Physical Review E 73 (2006) 016702.

[99] A. Tartakovsky, P. Meakin, Modeling of surface tension and contact angles with smoothed particle hydrodynamics, Physical Review E 72 (2005) 026301.

[100] G. Zhou, W. Ge, J. Li, A revised surface tension model for macro-scale particle methods, Powder Technology 183 (2008) 21-26.

[101] A.M. Aly, M. Asai, Y. Sonda, Modelling of surface tension force for free surface flows in ISPH method, International Journal of Numerical Methods for Heat \& Fluid Flow 23 (2013) 479-498.

[102] J.J. Monaghan, Smoothed particle hydrodynamics, Annual Review of Astronomy and Astrophysics 30 (1992) 543-574.

[103] A.M. Tartakovsky, P. Meakin, T.D. Scheibe, R.M.E. West, Simulations of reactive transport and precipitation with smoothed particle hydrodynamics, Journal of Computational Physics 222 (2007) 654-672.

[104] J. Monaghan, Smoothed particle hydrodynamics and its diverse applications, Annual Review of Fluid Mechanics 44 (2012) 323-346.

[105] J. Fang, A. Parriaux, M. Rentschler, C. Ancey, Improved SPH methods for simulating free surface flows of viscous fluids, Applied Numerical Mathematics 59 (2009) 251-271.

[106] J.P. Morris, P.J. Fox, Y. Zhu, Modeling low Reynolds number incompressible flows using SPH, Journal of Computational Physics 136 (1997) 214-226.

[107] G.-R. Liu, M.B. Liu, Smoothed Particle Hydrodynamics: a meshfree particle method, World Scientific2003. 
[108] A.P. Lima, A.S. Martins, J.S. Sá Martins, Lennard-Jones binary fluids: A comparative study between the molecular dynamics and Monte Carlo descriptions of their structural properties, Physica A: Statistical Mechanics and its Applications 391 (2012) 4281-4289.

[109] G.M. Torrie, J.P. Valleau, Monte Carlo study of a phase-separating liquid mixture by umbrella sampling, The Journal of Chemical Physics 66 (1977) 14021408.

[110] S.J. Cummins, M. Rudman, An SPH projection method, Journal of Computational Physics 152 (1999) 584-607.

[111] S. Shao, E.Y. Lo, Incompressible SPH method for simulating Newtonian and non-Newtonian flows with a free surface, Advances in Water Resources 26 (2003) 787-800.

[112] C. Heusser, Conjugate gradient-type algorithms for a finite-element discretization of the Stokes equations, Journal of Computational and Applied Mathematics 39 (1992) 23-37.

[113] S. Adami, X. Hu, N. Adams, A generalized wall boundary condition for smoothed particle hydrodynamics, Journal of Computational Physics 231 (2012) 7057-7075.

[114] H. Takeda, S.M. Miyama, M. Sekiya, Numerical simulation of viscous flow by smoothed particle hydrodynamics, Progress of Theoretical Physics 92 (1994) 939960.

[115] G. Taylor, The formation of emulsions in definable fields of flow, Proceedings of the Royal Society of London. Series A 146 (1934) 501-523.

[116] V. Sibillo, G. Pasquariello, M. Simeone, V. Cristini, S. Guido, Drop deformation in microconfined shear flow, Physical Review Letters 97 (2006) 054502.

[117] J. Han, G. Tryggvason, Secondary breakup of axisymmetric liquid drops. I. Acceleration by a constant body force, Physics of Fluids 11 (1999) 3650-3667.

[118] W.M. Saltzman, W.L. Olbricht, Building drug delivery into tissue engineering, Nature reviews. Drug Discovery 1 (2002) 177.

[119] A. Jahn, W.N. Vreeland, M. Gaitan, L.E. Locascio, Controlled vesicle selfassembly in microfluidic channels with hydrodynamic focusing, Journal of the American Chemical Society 126 (2004) 2674-2675.

[120] X. He, M. Aizenberg, O. Kuksenok, L.D. Zarzar, A. Shastri, A.C. Balazs, J. Aizenberg, Synthetic homeostatic materials with chemo-mechano-chemical selfregulation, Nature 487 (2012) 214.

[121] P. ZNIDARŠIC-PLAZL, Enzymatic microreactors utilizing non-aqueous media, Chimica Oggi-Chemistry Today 32 (2014) 1. 


\section{Supplementary Information}

\section{Investigating the parameter space for a droplet in a confined linear shear field}

Figure S1 shows a schematic of the model configuration used when considering a droplet in a confined linear shear field. The droplet, which is initially circular, is located at the centre of a channel of width, $H$, confined by walls that move at equal speed, $U$, but opposite direction. The walls are modelled by layers of SPH particles as illustrated in the insert at the top wall. For Capillary numbers below a critical value, the droplet deforms into an ellipsoid of dimensions $a$ and $b$ along the major and minor axes, and tilts at an angle $\varphi$ to the direction of flow - such a case is illustrated in Figures S2 and S3. Beyond the critical Capillary number, as illustrated by the case shown in Figure S4, the droplet deforms beyond the ellipsoidal shape shown here and ruptures to form two, smaller droplets.

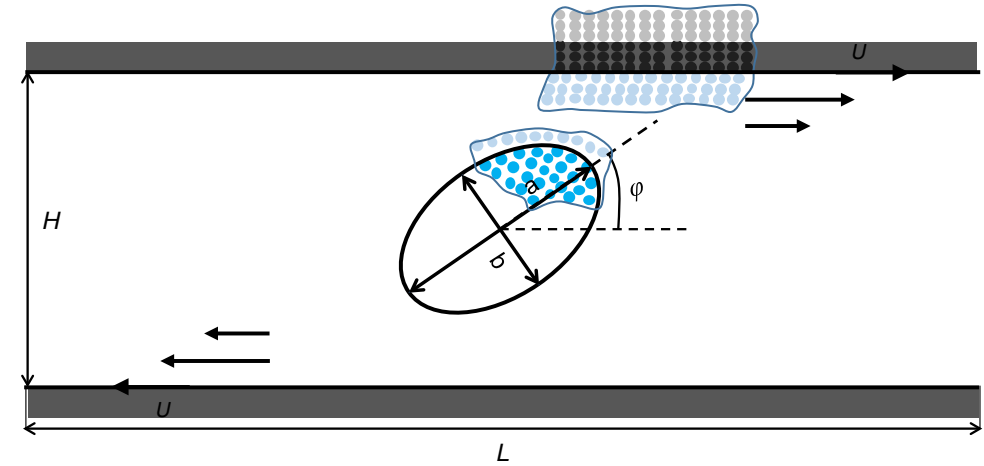

Fig. S1 Schematic of the model used when studying a droplet in a confined shear field.

Figure S2 shows a contour plot of fluid colour for a condition where the droplet significantly deforms from its initial circular shape but does not rupture. The contour behaviour at the leading and tail ends of the droplet reflect the small number of SPH particles of the droplet colour rather than penetration of the continuous phase into the droplet. Comparison of the colour variation at the interface with the support size of the SPH kernel (shown as a white bar in the figure) shows the new method can produce a sharp interface even when significant deformation occurs.

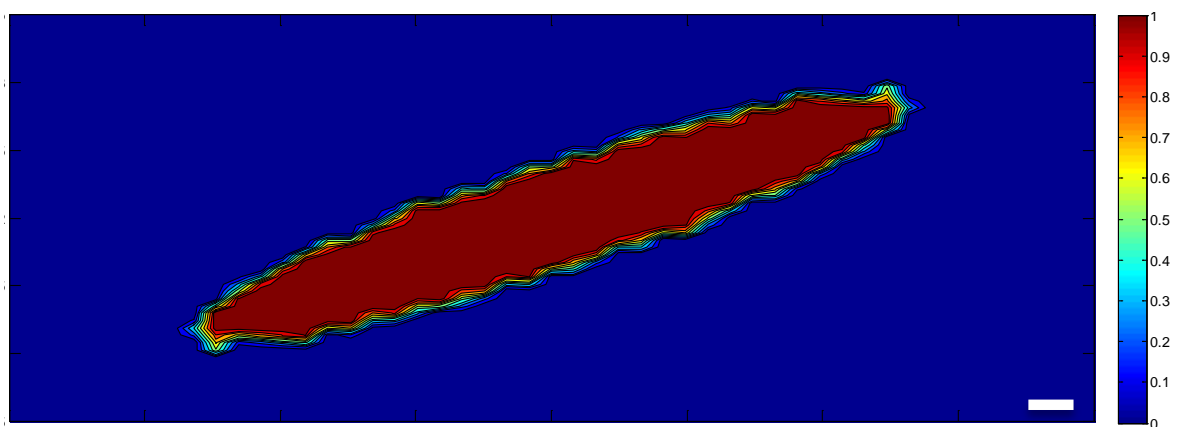

Fig. S2 Contour plot showing the spatial variation of the fluid colour from that of the dispersed (red) to the continuous phase (blue) for a confined droplet at steady state where $\mathrm{Ca}=\mu R G / \gamma=$ $0.2, \operatorname{Re}=\rho R^{2} G / \mu=0.1, \lambda=1, D / H=0.5, N_{p}=4961, L_{0}=1.0 \times 10^{-5}$ and $h=1.2 \times L_{0}$. The radius of the kernel size $(3 h)$ is shown as a solid white line in the bottom right hand corner. 
Figure S3 illustrates how the velocity field and droplet shape changes through time for a typical set of conditions under which the droplet ruptures into two daughter droplets. The shear across the droplet induces it to elongate and rotate gradually. This in turn leads to a distortion of the velocity field around the droplet as illustrated by the colour plots of the wider velocity field shown on the lefthand side of the figure. As seen in the close-ups of the droplets in the right hand of Figure S3, the flow around the droplet also leads to a circulatory flow within the droplet. The velocity vector plate for $t^{*}=9$ reveals very clearly the segregation of the velocity field within the droplet that presages its rupture soon thereafter.
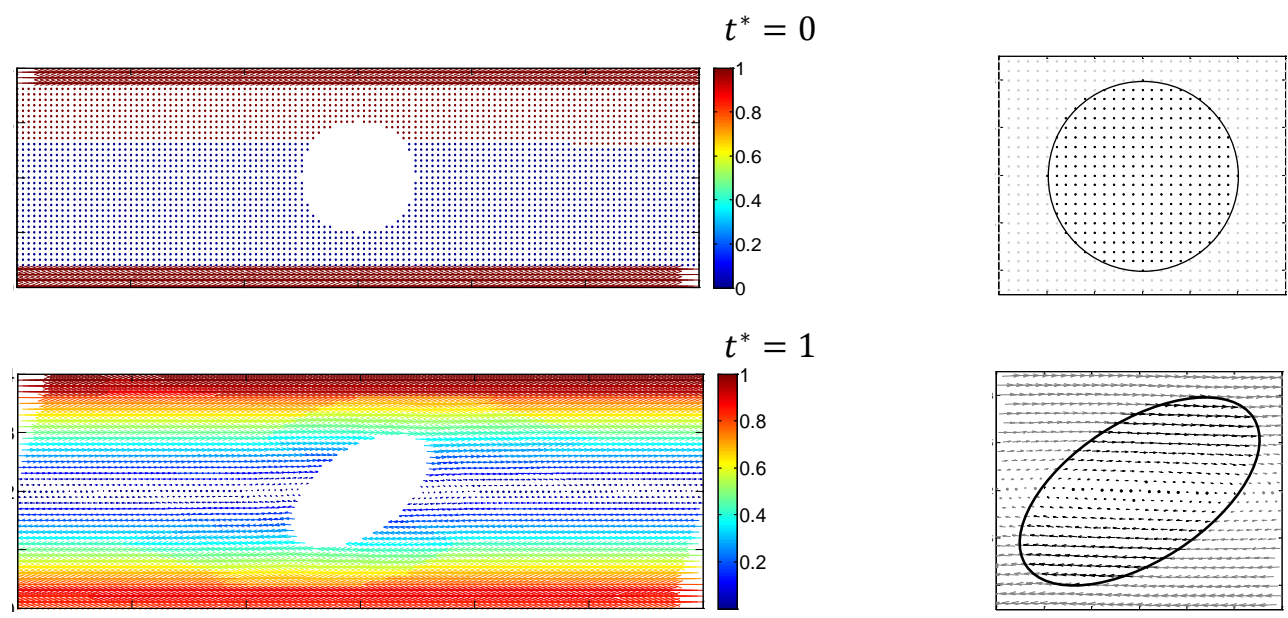

$$
t^{*}=5
$$
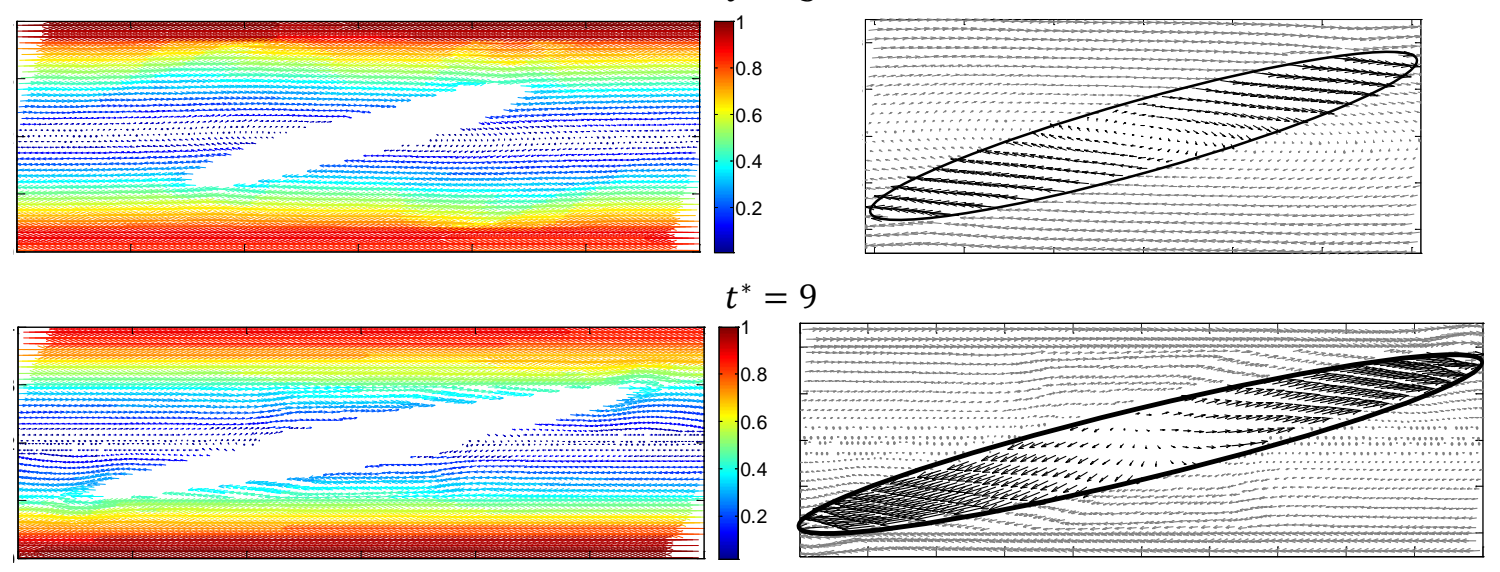

$t^{*}=12$
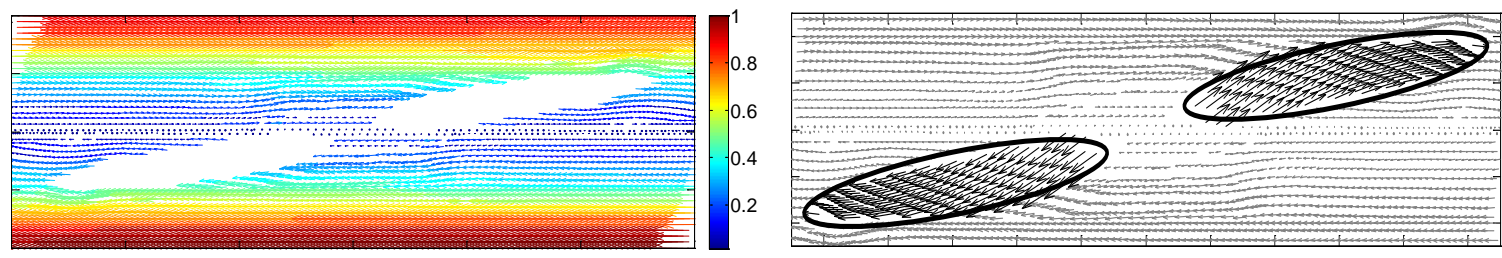

Fig. S3 Velocity field \& droplet morphology through dimensionless time, $t^{*}=G t$, for a confined droplet where $\mathrm{Ca}=0.5, \mathrm{Re}=0.1, \lambda=1, D / H=0.5, N_{p}=4961, L_{0}=1.0 \times 10^{-5}$, and $h=1.2 \times L_{0}$. 
Figure S4 illustrates how the velocity field and droplet shape changes through time for the same conditions associated with Figure S2.
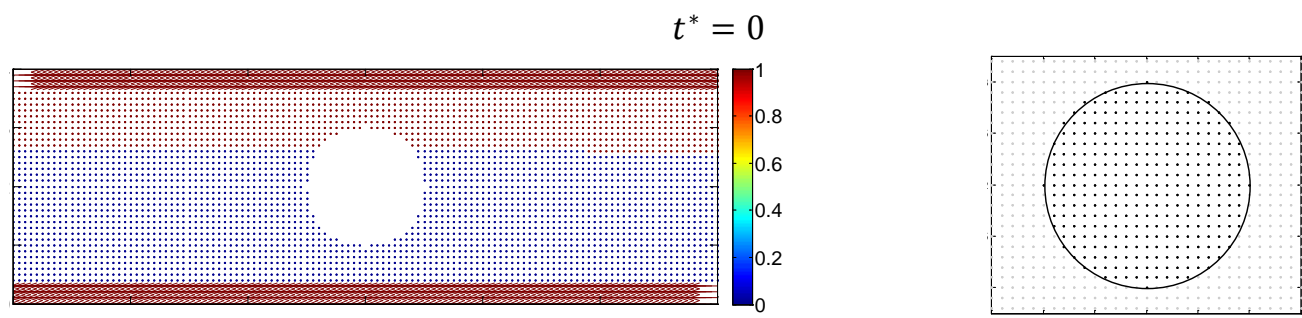

$t^{*}=1$
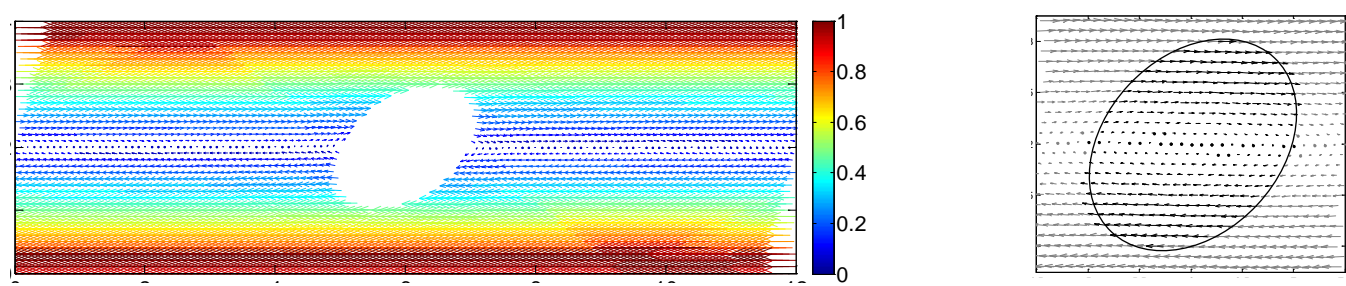

$$
t^{*}=3
$$
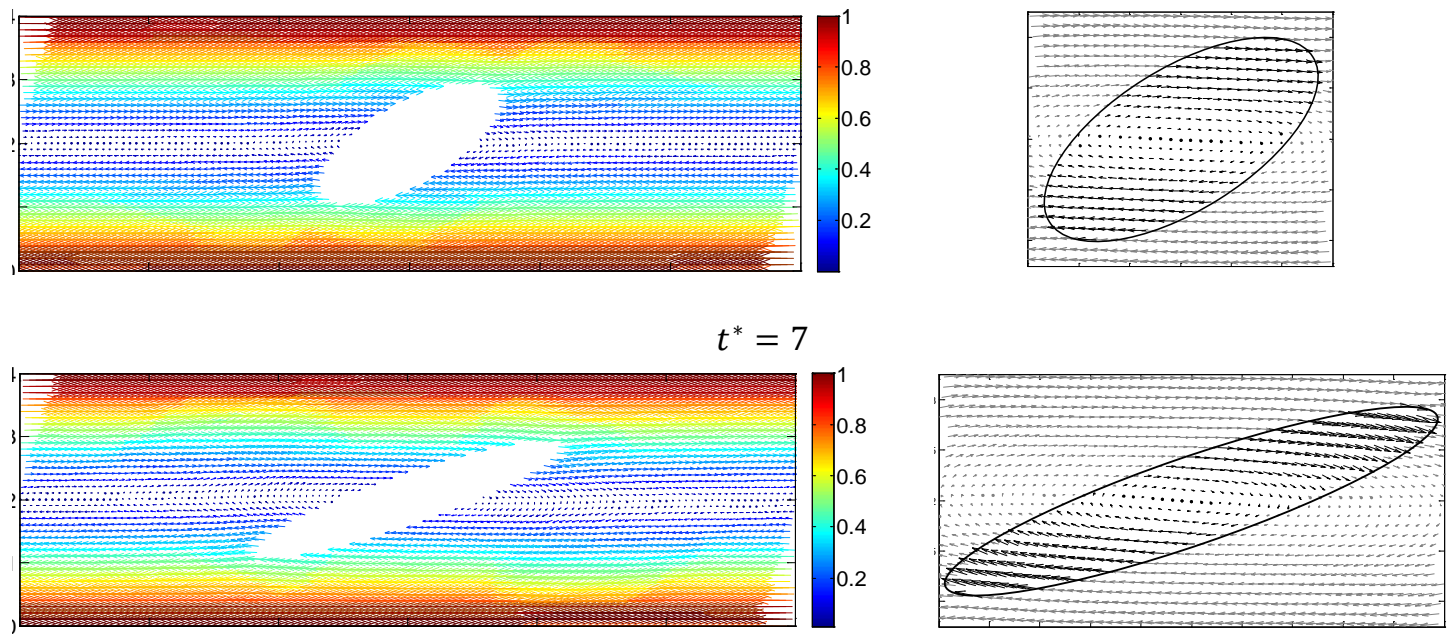

$$
t^{*}=10
$$

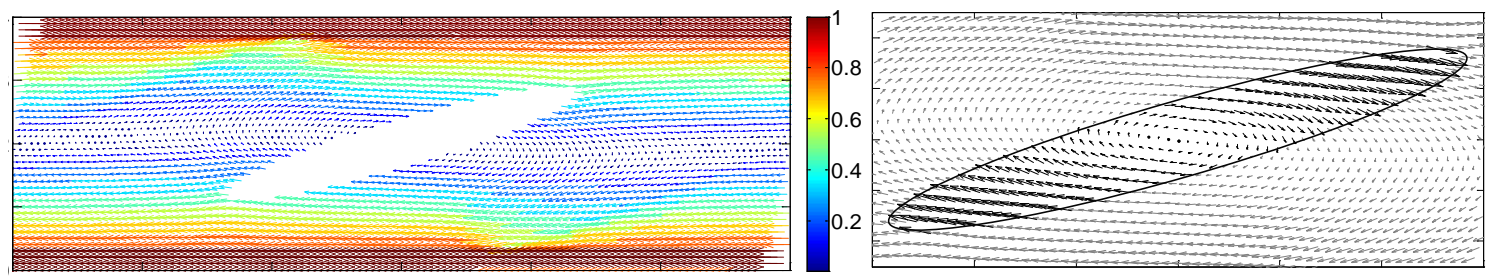

Fig. S4 Velocity field \& droplet morphology through dimensionless time, $t^{*}=G t$, for a confined droplet where $\mathrm{Ca}=\mu R G / \gamma=0.2, \operatorname{Re}=\rho R^{2} G / \mu=0.1, \lambda=1, D / H=0.5, N_{p}=4961, L_{0}=$ $1.0 \times 10^{-5}$ and $h=1.2 \times L_{0}$. 


\section{Investigating the parameter space for a droplet descending in a stationary column of less-dense liquid}

We present here a further result to demonstrate the capacity the new method provides to investigate the parameter space associated with a droplet descending in a stationary column of less-dense liquid. Figure S5 illustrates the effect that the Eötvös Number has on the droplet behaviour. In both cases, the droplet rapidly accelerates before achieving a steady state decent speed with some degree of overshoot in between. Increase in Eo sees a greater overshoot followed by an undershoot before it reaches steady-state. This difference in behaviour seen here is understood by considering the extent of change in the droplet shape during descent, which is illustrated in Figures S5(b) and (c). These results are in agreement with the shape regime maps developed by Han and Tryggvason [117].

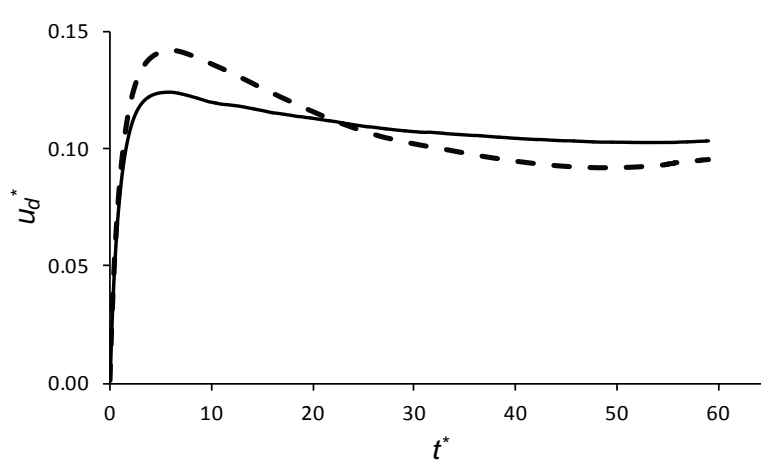

(a)

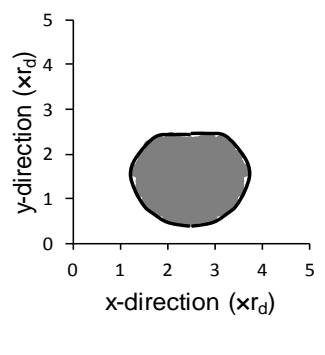

(b)

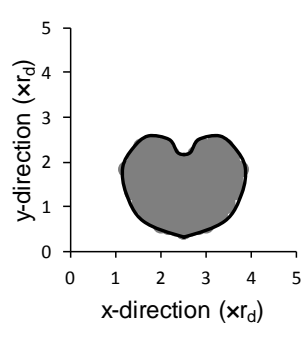

(c)

Fig. S5 Droplet descending in a stationary column of less-dense liquid: (a) Variation of droplet descent speed with time as predicted by SPH for $\eta=1.15, O h_{d}=0.23$ and $O h_{o}=1.25$ with $E o=$ 24 (solid line) and $E o=144$ (broken line); (b) SPH particle positions at steady state showing final droplet shape for $E o=24$; and (c) SPH particle positions at steady state showing final droplet shape for $E o=144$. In (b) and (c) the black outline demarks the interface between the two phases. 